\title{
Durability of Response to Tocilizumab Therapy in Rheumatoid Arthritis: Data from the US-Based Corrona Rheumatoid Arthritis Registry
}

\author{
Dimitrios A. Pappas · Taylor Blachley · Jennie H. Best • \\ Steve Zlotnick • William G. Reiss · Kelechi Emeanuru • \\ Joel M. Kremer
}

Received: November 25, 2020 / Accepted: January 23, 2021 / Published online: February 25, 2021

(C) The Author(s) 2021

\section{ABSTRACT}

Introduction: Understanding the durability of response to treatment and factors associated with failure to maintain response in a real-world setting can inform treatment decisions for patients with rheumatoid arthritis (RA). The aim of this study was to analyze durability of response to tocilizumab (TCZ) and factors associated with durability among US patients with RA in routine clinical practice.

Methods: TCZ initiators in the Corrona RA Registry were included. Durability of response was defined as maintaining continuous TCZ treatment and either an improvement of at least minimum clinically important difference (MCID) in Clinical Disease Activity Index (CDAI) score or low disease activity (LDA). Secondary analyses included patients treated with intravenous (IV) TCZ and excluded those who discontinued TCZ without reporting reasons for discontinuation. Durability was calculated with Kaplan-Meier survival analysis. Cox

D. A. Pappas ( $₫) \cdot$ T. Blachley · K. Emeanuru •

J. M. Kremer

Corrona, LLC, Waltham, MA, USA

e-mail: dpappas@corrona.org

J. H. Best · S. Zlotnick · W. G. Reiss

Genentech, Inc., South San Francisco, CA, USA proportional hazards modeling identified factors associated with durability.

Results: Among 1789 TCZ initiators, 466, 272, and 162 were persistent (with or without durable response) with follow-up visits at 1,2, and 3 years, respectively. Median MCID durability of response in CDAI was $>50 \%$ after 36 months overall, 26 months for TCZ-IV, and $>50 \%$ after 36 months for those with known reasons for discontinuation; longer durability was associated with increased duration of RA and higher baseline CDAI score and shorter durability with history of malignancy and history of diabetes. Median LDA durability of response was 13.0 months overall, for TCZ-IV, and for those with known reasons for discontinuation; shorter durability was associated with history of malignancy, history of diabetes, and higher baseline CDAI score.

Conclusions: Median durability of response to TCZ in RA was $>3$ years when defined as maintenance of MCID in CDAI score and $>1$ year with the more stringent criteria of maintenance of LDA.

Trial Registration: ClinicalTrials.gov identifier, NCT01402661

Keywords: Biological therapies; Biologics; Durability of response; Real-world data; Rheumatoid arthritis; Tocilizumab

J. M. Kremer

Albany Medical College and the Center for

Rheumatology, Albany, NY, USA 


\section{Key Summary Points}

\section{Why carry out this study?}

Using real-world data to analyze the durability of response to treatment and factors associated with failure to maintain response can inform treatment decisions for patients with rheumatoid arthritis (RA); for these patients, less is known about the durability of response to biologic therapies.

This study used real-world data to analyze the durability of response to the biologic therapy tocilizumab (TCZ) and factors associated with durability among US patients with RA.

Durability of response was defined as maintaining both continuous TCZ therapy and concurrent continuous response to therapy.

\section{What has been learned from the study?}

Among patients with RA in routine clinical practice, median durability of response to TCZ was $>3$ years when measured as maintenance of minimum clinically important difference in Clinical Disease Activity Index score; even with the more stringent criteria of maintenance of low disease activity, median TCZ durability of response was $>1$ year.

These results may help inform treatment decisions for patients with RA.

\section{DIGITAL FEATURES}

This article is published with digital features, including a summary slide, to facilitate understanding of the article. To view digital features for this article go to https://doi.org/10.6084/ m9.figshare.13622894.

\section{INTRODUCTION}

Rheumatoid arthritis (RA) is a systemic inflammatory disease affecting the joints; it results in chronic joint swelling, stiffness, and pain and can lead to joint damage and disability [1]. The first line of treatment for patients with RA is conventional synthetic disease-modifying antirheumatic drugs (csDMARDs); however, to achieve the recommended goal of low disease activity (LDA) or remission, successive therapy changes are often needed [2]. Patients who do not achieve disease control with csDMARDs alone are usually treated with targeted synthetic DMARDs (tsDMARDs) or biologics [2]. There are now five families of molecular targets for RA treatment, several of which have multiple therapy options [2]. Although the efficacy of biologics in patients with RA has been extensively studied [3], less is known about the durability or maintenance of response to biologics. Understanding the durability of response to treatment and factors associated with failure to maintain response in a real-world setting can inform treatment decisions for patients with RA.

There is a critical difference between durability of response to therapy and persistency of therapy. Many studies have addressed the persistency of biologics, which is often measured as the time from treatment initiation until treatment discontinuation [4, 5]. This has been considered a proxy to therapy effectiveness, and the assumption is frequently made that the patient is responding to therapy as long as the therapy is not stopped. Maintaining a concurrent response to treatment is not taken into account in analyses evaluating persistency of biologics, csDMARDs, and tsDMARDs. In the present analysis, we evaluated the durability of response, which was defined as persistence on therapy while maintaining concurrent response to therapy. To the best of our knowledge, this approach has not previously been published for biologic treatment in patients with RA.

The present study evaluated the durability of response to tocilizumab (TCZ), a monoclonal antibody against the interleukin- 6 receptor, in US patients with RA initiating TCZ in routine 
clinical practice. Factors affecting durability of response were also assessed. TCZ is approved for the treatment of RA in patients with an inadequate response to DMARDs [6]; TCZ may be administered as monotherapy or in combination with other csDMARDs, and clinical studies have demonstrated that both strategies effectively reduce disease activity [7-14]. While studies have demonstrated the long-term safety, efficacy, and real-world persistence of TCZ therapy, data on the durability of response to TCZ are lacking $[5,15,16]$.

\section{METHODS}

\section{Study Setting}

The Corrona RA registry is an ongoing independent, prospective, observational cohort of patients with RA (NCT01402661) [17, 18]. Patients are recruited from 193 private and academic practice sites across 42 states in the United States, with 827 participating rheumatologists. As of March 31, 2020, data on 54,453 patients with RA had been collected. Corrona's database includes information from 414,251 patient visits and 199,111 patient-years of follow-up observation time. The mean duration of patient follow-up is 4.49 years (median, 3.2 years). Ethics approval for this study was obtained from a central institutional review board (IRB; New England Independent Review Board 120160610). For academic investigative sites that did not receive a waiver to use the central IRB, full board approval was obtained from the respective governing IRBs, and documentation of approval was submitted to the sponsor prior to initiating any study procedures. All procedures performed in studies involving human participants were in accordance with the ethical standards of the institutional and/or national research committee and with the 1964 Helsinki Declaration and its later amendments or comparable ethical standards. Informed consent was obtained from all individual participants included in the study.

\section{Study Population}

TCZ-naïve patients aged $\geq 18$ years enrolled in the Corrona RA registry who initiated TCZ (subcutaneous or intravenous [IV]) after January 1, 2010, had baseline Clinical Disease Activity Index (CDAI) scores available, and had $\geq 1$ follow-up registry visit-regardless of continuation of therapy-were included. Patients who discontinued TCZ after less than 1 month were not considered TCZ initiators because of the possibility of prescriptions being unfilled or never started. Data analyzed were collected from physician and patient questionnaires that were completed during routine clinical encounters over the study period. The Corrona Registry has been previously described in detail elsewhere [17]. Data collected during participation in the registry include patient demographics, clinical characteristics, history of comorbidities, current and prior medication use, clinical disease activity measures, and patient-reported outcomes [17]. Durability of response at 1,2, and 3 years was assessed in patients who were persistent on medication and who had a follow-up visit at these time points so that disease activity could be measured. Persistency at 1, 2, or 3 years did not require follow-up visits (or disease activity evaluation) at these time points, as a later visit could be used to infer persistency at earlier timepoints (i.e., a patient who had a follow-up visit at year 3 and was receiving the medication, but who did not have a visit at year 2 , was considered persistent at years 2 and 3 ).

\section{Study Design and Outcomes}

This observational study evaluated the durability of response and factors associated with decreased durability in patients with RA initiating TCZ. Durability of response was defined as maintaining continuous TCZ and 1 of the following: (a) continuous maintenance of at least a minimum clinically important difference (MCID) in CDAI score, defined as an improvement in CDAI score of $\geq 2$ if the baseline was $\leq 10, \geq 6$ if the baseline was $>10$ to $\leq 22$, and $\geq 11$ if the baseline was $>22$ [19] or (b) continuous maintenance of at least LDA 
$(\mathrm{CDAI} \leq 10)$. Patient response was no longer durable upon the first discontinuation of TCZ or the first failure to maintain MCID in CDAI score or LDA. The primary outcome was the durability of response up to 36 months and factors associated with decreased durability in all TCZ initiators. Secondary analyses estimated durability after (a) including only those who were treated with TCZ-IV or (b) excluding patients without a reported reason for discontinuing TCZ (patients with safety- or efficacy-related reasons for discontinuation were defined as failing to maintain durability, whereas nonmedical reasons for discontinuation [e.g., insurance coverage] were censored at the time of discontinuation).

\section{Statistical Analyses}

Baseline characteristics were summarized using means and SDs for continuous variables and frequencies and percentages for categorical variables. Kaplan-Meier product-limit estimates of survival were used to estimate durability.
Factors associated with durability were identified through Cox proportional hazards modeling.

\section{RESULTS}

A total of 1789 TCZ initiators with $\geq 1$ followup visit were identified (Fig. 1). The mean (SD) time between follow-up visits (after TCZ initiation) was 6.7 (4.4) months. Among the 1789 TCZ initiators, $81.0 \%$ were female, $85.0 \%$ were white, and $75.4 \%$ were overweight or obese (Table 1). The mean (SD) age was 58.5 (12.6) years, duration of RA was 12.0 (9.6) years, and CDAI score was 23.2 (14.2). Most patients (93.4\%) had previously received biologics, and $67.4 \%$ had received $\geq 2$ prior biologics. Overall, $38.1 \%$ initiated TCZ as monotherapy. Secondary analyses included 1284 patients who initiated TCZ-IV and 1303 patients with known reasons for discontinuing TCZ (patients with safety- or efficacy-related reasons for discontinuation were defined as failing to maintain

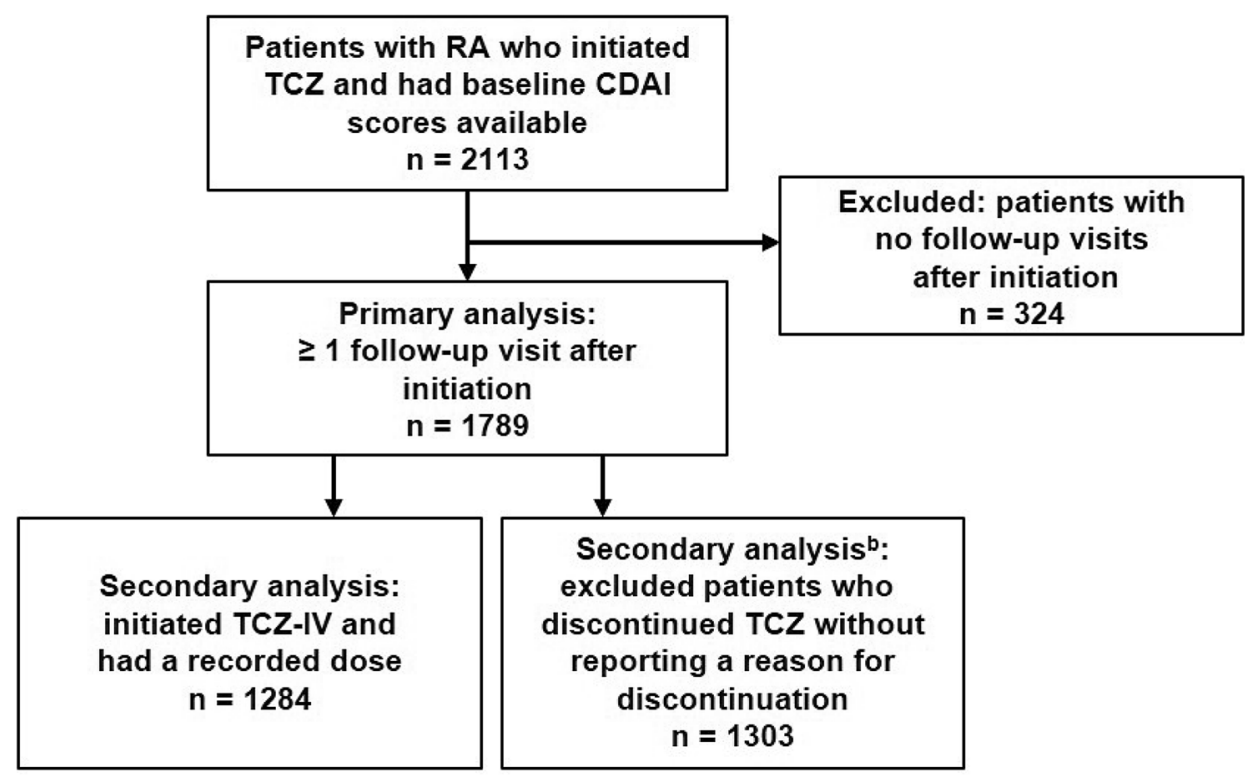

Fig. 1 Selection of eligible patients for analysis. ${ }^{a} C D A I$ Clinical Disease Activity Index, $I V$ intravenous, $R A$ rheumatoid arthritis, $T C Z$ tocilizumab. ${ }^{a}$ Patients selected using the January 31, 2019, version of the Corrona RA database. ${ }^{b}$ Patients with safety- or efficacy-related reasons for discontinuation were defined as failing to maintain durability, whereas nonmedical reasons for discontinuation [e.g., insurance coverage] were censored at the time of discontinuation 
Table 1 Baseline patient characteristics

TCZ Initiators $(N=1789)$

Female, $n(\%)$

1449 (81.0)

Age, mean (SD), years

$58.5(12.6)$

Duration of RA, mean (SD), years

$12.0(9.6)$

White, $n$ (\%)

$1512(85.0)$

Previous or current smoker, $n(\%)^{\mathrm{a}}$

$873(49.1)$

BMI category, $n$ (\%)

Underweight/normal weight $\left(<25 \mathrm{~kg} / \mathrm{m}^{2}\right)$

$439(24.5)$

Overweight ( $\geq 25$ to $<30 \mathrm{~kg} / \mathrm{m}^{2}$ )

$524(29.3)$

Obese $\left(>30 \mathrm{~kg} / \mathrm{m}^{2}\right)$

$825(46.1)$

Insurance, $n(\%)^{\mathrm{b}}$

None

$11(0.6)$

Private

$1322(73.9)$

Medicaid

$92(5.1)$

Medicare

$681(38.1)$

History of comorbid conditions, $n$ (\%)

Hypertension

$596(33.3)$

Diabetes

$185(10.3)$

Malignancy ${ }^{\mathrm{c}}$

$110(6.1)$

$\mathrm{CVD}^{\mathrm{d}}$

$736(41.1)$

Medication history

Prior csDMARD use, $n(\%)^{e}$

0

87 (4.9)

1

$616(34.4)$

$\geq 2$

$1086(60.7)$

Prior TNFi use, $n(\%)^{\mathrm{e}}$

0

$209(11.7)$

1

$673(37.6)$

$\geq 2$

907 (50.7)

Prior non-TNFi use, $n(\%)^{\mathrm{e}}$

0

$867(48.5)$ 
Table 1 continued

TCZ Initiators $(N=1789)$

1

$\geq 2$

Prior biologic use, $n(\%)^{\mathrm{e}}$

0

1

466 (26.0)

$\geq 2$

1205 (67.4)

Concomitant medication, $n$ (\%)

Monotherapy

Combination with csDMARDs

1107 (61.9)

Prednisone use, $n$ (\%)

Disease activity

Tender joint count (0-28), mean (SD)

Swollen joint count (0-28), mean (SD)

Physician global assessment (0-100), mean (SD)

Patient global assessment (0-100), mean (SD)

CDAI (0-76), mean (SD)

Patient pain (0-100), mean (SD)

mDAS (1.69-8.75), mean (SD)

mHAQ (0-3), mean (SD)

Patient fatigue (0-100), mean (SD)

Morning stiffness, $n$ (\%)

$1586(89.3)$

$B M I$ body mass index, $C D A I$ Clinical Disease Activity Index, csDMARD conventional synthetic disease-modifying antirheumatic drug, $C V D$ cardiovascular disease, $m D A S$ modified Disease Activity Score, $m H A Q$ modified Health Assessment Questionnaire, $R A$ rheumatoid arthritis, $T C Z$ tocilizumab, $T N F i$ tumor necrosis factor inhibitor

a $n=1777$

b Totals may not add up to $100 \%$ because patients may have $\geq 1$ type of insurance

c Malignancy includes breast cancer, lung cancer, lymphoma, skin cancer (melanoma and squamous cell), and other cancers

d History of CVD includes history of cardiac revascularization procedure (coronary artery bypass grafting, stent, angioplasty), ventricular arrhythmia, cardiac arrest, myocardial infarction, acute coronary syndrome, unstable angina, other coronary artery disease, congestive heart failure (with and without hospitalization), stroke, transient ischemic attack, other cardiovascular, deep vein thrombosis, peripheral arterial disease, pulmonary embolism, and carotid artery disease

e Including past and current therapies. csDMARDs: methotrexate, hydroxychloroquine, leflunomide, sulfasalazine, azathioprine, minocycline, and cyclosporine. TNFis: adalimumab, etanercept, certolizumab pegol, golimumab, and infliximab. Non-TNFis: sarilumab, abatacept, and rituximab (tocilizumab excluded). Biologics: adalimumab, etanercept, certolizumab pegol, golimumab, infliximab, sarilumab, abatacept, rituximab, and tofacitinib 


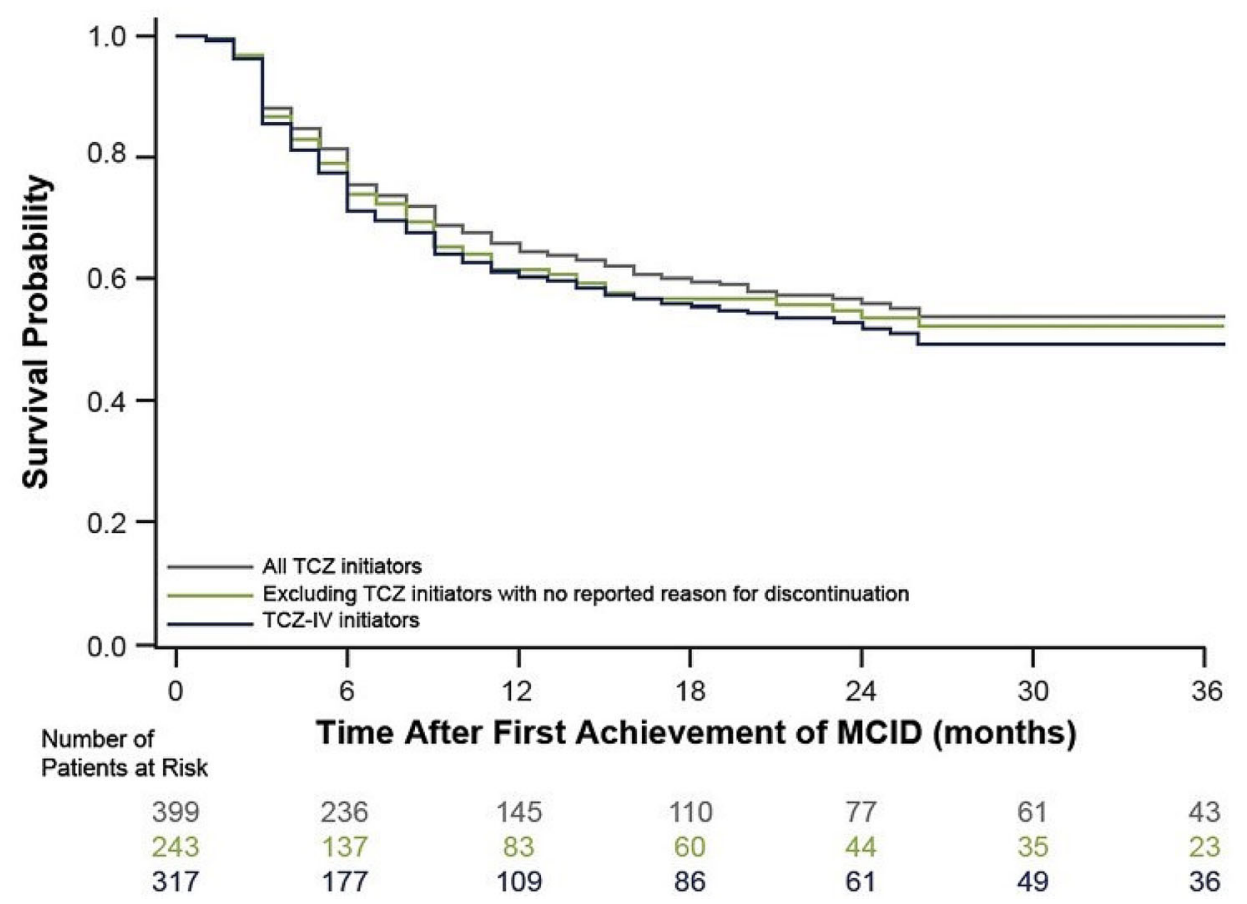

Fig. 2 Kaplan-Meier plots of MCID durability of response among patients who achieved MCID. $I V$ intravenous, $M C I D$ minimum clinically important difference, $T C Z$ tocilizumab

durability, whereas nonmedical reasons for discontinuation [e.g., insurance coverage] were censored at the time of discontinuation). At 1 , 2 , and 3 years, 861, 483, and 298 patients were persistent (on therapy with or without durable response), respectively. Among these patients, 466,272 , and 162 had follow-up visits at 1,2 , and 3 years, respectively.

\section{MCID Durability of Response}

With the Kaplan-Meier estimate of the survival function among all TCZ initiators who achieved MCID in CDAI, durability of response, measured by continuous TCZ and maintenance of MCID, remained $>50 \%$ after 36 months of follow-up (Fig. 2); the precise median (95\% CI) MCID durability was not estimable (NE) because the survival rate was $>50 \%$. For these patients, the proportion $(95 \% \mathrm{CI})$ maintaining MCID durability at 1,2 , and 3 years was $64.4 \%$ (59.2-69.6\%), 56.0\% (50.0-62.0\%), and 51.8\% (44.7-58.9\%), respectively. For TCZ-IV initiators who achieved MCID in CDAI score, median
(95\% CI) MCID durability of response was 26 (17-NE) months (Fig. 2). The estimation of the upper confidence limit was NE because, due to a lack of events, the survival estimate did not drop to a low enough level prior to the end of study follow-up. At 1, 2, and 3 years, the proportion of patients $(95 \% \mathrm{CI})$ maintaining MCID durability was $60.2 \% \quad(54.2-66.2 \%), \quad 51.9 \%$ (45.2-58.6\%), and 47.4\% (39.8-55.0\%), respectively, among these patients. Among patients with known reasons for discontinuation who achieved MCID, median (95\% CI) MCID durability of response was 45 (16-NE) months (Fig. 2). For these patients, the proportion (95\% CI) maintaining MCID durability was $60.8 \%$ (53.8-67.7\%), 53.5\% (45.7-61.3\%), and 49.0\% $(39.3-58.7 \%)$ at 1,2 , and 3 years, respectively.

Among all TCZ initiators who achieved MCID, longer MCID durability of response (hazard ratio [HR]; 95\% CI) was associated with increased duration of RA (0.91 [0.83-0.99]; $p=0.04)$ and higher baseline CDAI score $(0.72$ [0.60-0.87]; $p=0.001$ ); factors associated with shorter MCID durability included history of malignancy (2.88 [1.57-5.27]; $p=0.001)$ and 
A

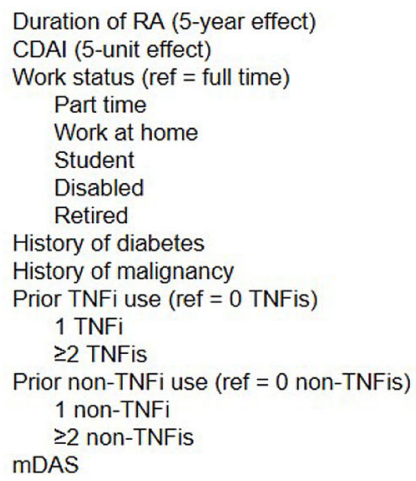

All TCZ Initiators
B

Duration of RA (5-year effect) CDAI (5-unit effect) $\mathrm{TCZ}$ dose $(8 \mathrm{mg} / \mathrm{kg}$ vs $4 \mathrm{mg} / \mathrm{kg}$ ) History of diabetes History of malignancy Prior TNFi use (ref $=0$ TNFis) $1 \mathrm{TNFi}$

$$
\geq 2 \text { TNFis }
$$

Prior biologic use (ref $=0$ biologics) 1 biologic

2 biologics

$\geq 3$ biologics mDAS

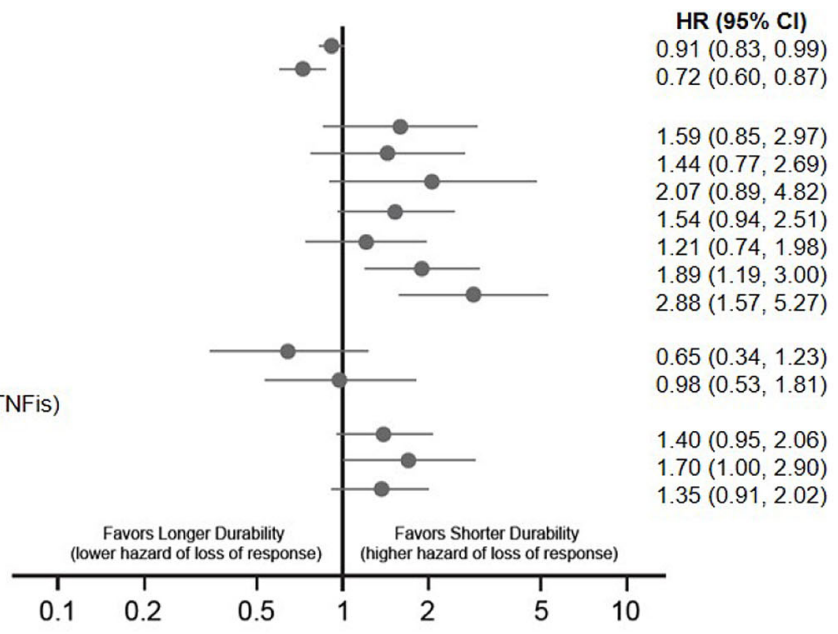

TCZ-IV Initiators

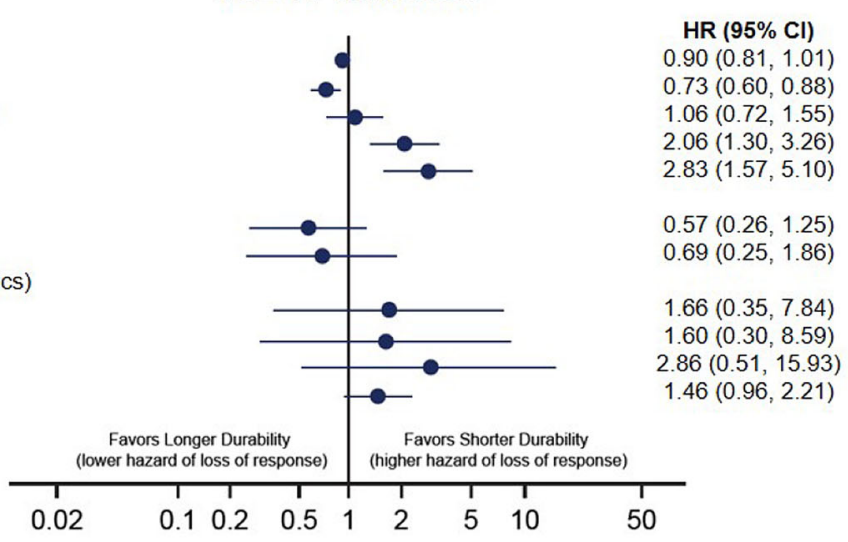

TCZ Initiators Excluding Those with No Reported Reason for Discontinuation
Duration of RA (5-year effect) CDAl (5-unit effect)

Work status (ref $=$ full time) Part time Work at home

Student

Disabled Retired

History of diabetes

History of malignancy

Prior non-TNFi use (ref $=0$ non-TNFis) 1 non-TNFi

$$
\geq 2 \text { non-TNFis }
$$

Prior biologic use (ref $=0$ biologics) 1 biologic

2 biologics

$\geq 3$ biologics

Concomitant medication (ref = monotherapy) Combination with csDMARD

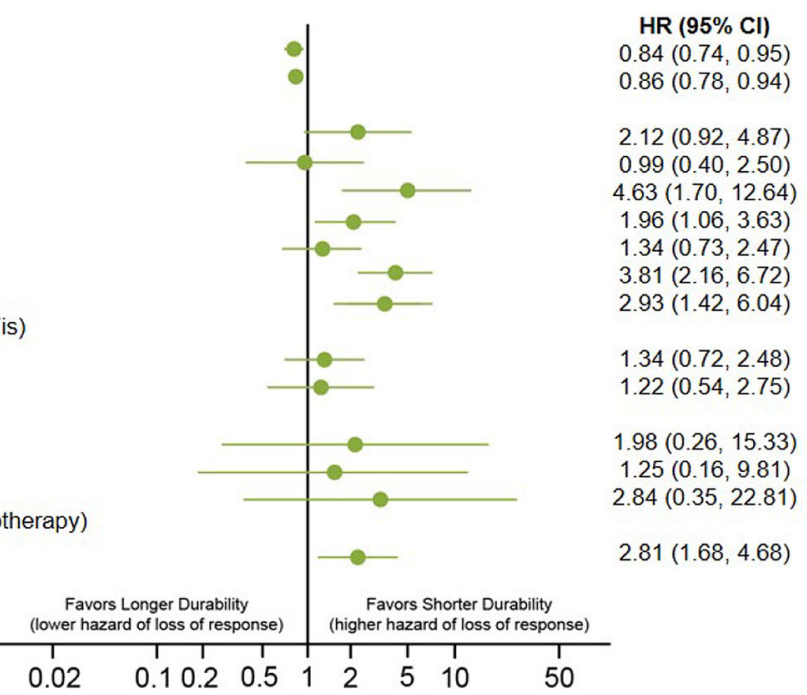


4Fig. 3 Estimated effects of covariates on MCID durability of response. $C D A I$ Clinical Disease Activity Index, csDMARD conventional synthetic disease-modifying antirheumatic drug, $I V$ intravenous, $M C I D$ minimum clinically important difference, $m D A S$ modified Disease Activity Score, $R A$ rheumatoid arthritis, Ref reference, $T C Z$ tocilizumab, $T N F i$ tumor necrosis factor inhibitor

history of diabetes (1.89 [1.19-3.00]; $p=0.01)$ (Fig. 3a). For TCZ-IV initiators, longer MCID durability (HR [95\% CI]) was associated with higher baseline CDAI score $(0.73$ [0.60-0.88]; $p=0.001)$, whereas history of malignancy $(2.83$ [1.57-5.10]; $p=0.001)$ and history of diabetes (2.06 [1.30-3.26]; $p=0.002$ ) were associated with shorter MCID durability (Fig. 3b). When including only patients with known reasons for discontinuation, longer MCID durability (HR [95\% CI]) was associated with increased duration of RA (0.84 [0.74-0.95]; $p=0.01)$ and higher baseline CDAI score (0.86 [0.78-0.94]; $p=0.001)$; factors associated with shorter MCID durability included history of malignancy (2.93
$[1.42-6.04] ; p=0.004)$ and history of diabetes (3.81 [2.16-6.72]; $p<0.0001$ ) (Fig. 3c).

\section{LDA Durability of Response}

With the Kaplan-Meier estimate of the survival function among all TCZ initiators who achieved LDA, the median (95\% CI) LDA durability of response, defined by continuous TCZ and maintenance of LDA, was 13.0 (12.0-20.0) months (Fig. 4). For these patients, the proportion $(95 \% \mathrm{CI})$ maintaining LDA durability at 1 , 2 , and 3 years was $52.3 \%(45.7-59.0 \%), 37.6 \%$ (30.2-45.0\%), and $27.3 \%$ (18.2-36.4\%), respectively. Among TCZ-IV initiators who achieved LDA, median (95\% CI) LDA durability was 13.0 (10.0-19.0) months (Fig. 4). At 1, 2, and 3 years, the proportion of patients (95\% CI) maintaining LDA durability was $50.8 \%(43.3-58.3 \%)$, $34.9 \%$ (26.6-43.1\%), and $26.0 \%(16.2-35.8 \%)$, respectively, among the TCZ-IV patients who achieved LDA. Among patients with known reasons for discontinuation who achieved LDA, median (95\% CI) LDA durability was 13.0 (9.0-29.0) months (Fig. 4). For these patients,

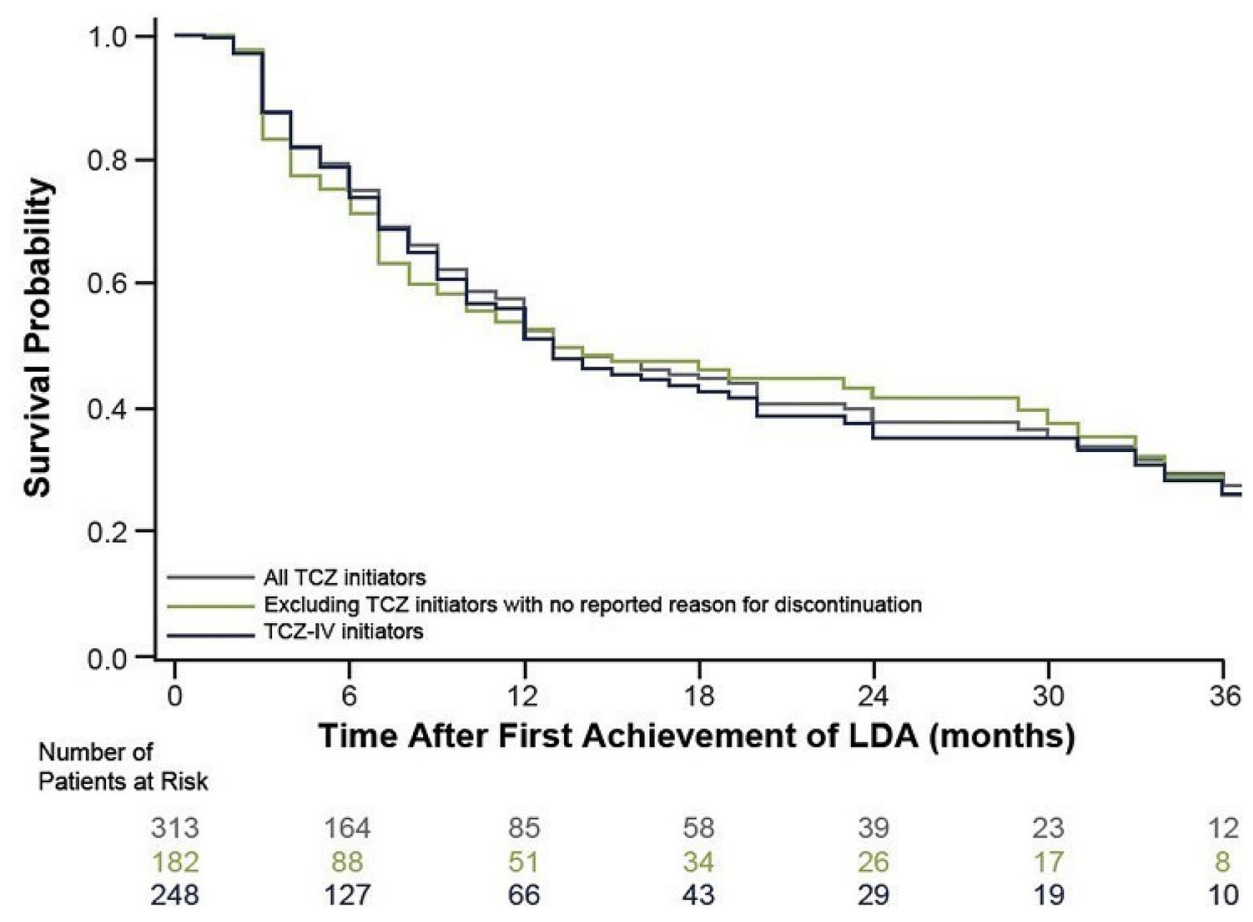

Fig. 4 Kaplan-Meier plots of LDA durability of response among patients who achieved LDA. $I V$ intravenous, $L D A$ low disease activity, $T C Z$ tocilizumab 
the proportion $(95 \% \mathrm{CI})$ maintaining LDA durability was $52.6 \% \quad(44.1-61.2 \%), \quad 41.5 \%$ (32.0-50.9\%), and $25.7 \%(13.7-37.6 \%)$ at 1,2 , and 3 years, respectively.

Among all TCZ initiators who achieved LDA, factors associated with shorter LDA durability (HR [95\% CI]) included history of malignancy (2.83 [1.66-4.82]; $p=0.0001)$, history of diabetes (1.69 [1.03-2.77]; $p=0.04)$, and higher baseline CDAI score (1.11 [1.05-1.17]; $p=0.001$ ) (Fig. 5a). For TCZ-IV initiators, shorter LDA durability (HR $[95 \% \mathrm{CI}]$ ) was associated with history of malignancy (2.92 [1.65-5.15]; $p=0.0002)$, history of diabetes (1.80 [1.08-3.00]; $p=0.02)$, and higher baseline CDAI score $(1.10$ [1.03-1.17]; $p=0.004)$ (Fig. 5b). When only patients with known reasons for discontinuation were included, factors associated with shorter LDA durability (HR [95\% CI]) included history of malignancy (2.57 [1.37-4.80]; $p=0.003$ ) and higher baseline CDAI score (1.14 [1.06-1.23]; $p=0.001)$ (Fig. 5c).

\section{DISCUSSION}

This study evaluated durability of response to TCZ therapy, defined as continuous therapy with concurrent maintenance of disease activity response, in US TCZ-naïve patients with RA in clinical practice. This study also assessed factors associated with durability. Durability of response was measured in two ways: (a) as continuous TCZ and maintenance of at least MCID in CDAI score or (b) as continuous TCZ and maintenance of at least LDA. Such an evaluation of therapy effectiveness, including both persistence on therapy as well as maintenance of concurrent response to treatment in a single outcome, has not to our knowledge previously been reported for biologic therapy in patients with RA. Multiple large registry studies have evaluated drug retention separately from change in disease activity measures over time $[20,21]$. Using the aforementioned definition of durability, the present study observed a median durability of response to TCZ therapy of $>3$ years when measured as maintenance of MCID; even with the more stringent criteria of maintenance of LDA, durability of response was $>1$ year.

Persistence on therapy, which has been studied for biologics in RA [4, 5], has been considered indirect evidence of response to treatment, but this is not always accurate because patients may continue treatment despite having uncontrolled disease activity $[22,23]$. In a behavioral intervention trial of treat-to-target care in RA, treatment was not accelerated despite disease activity measures not being met (CDAI > 10) at approximately $47 \%$ of patient visits, and barriers to accelerating treatment included patient reluctance and medication lag time [22]. Patient preference was also observed as a barrier to adjusting treatment in a randomized controlled study of implementing the treat-to-target approach in RA [23]. These studies demonstrated that persistence on therapy is not always sufficient to indicate effectiveness of medications and does not reflect continuous response of disease activity.

Persistency data reported in the literature do not account for concurrent response to treatment, as is done in the present study; this may explain discrepancies between results reported in the existing literature and what is reported here. A large, observational study in patients with RA in Canada found that, over a mean (SD) follow-up of 1.9 (1.6) years, $38.7 \%$ of patients discontinued biologics [24]. This included patients discontinuing for ineffectiveness (20.3\%), adverse events $(8.1 \%)$, and other reasons (10.3\%). A US administrative claims study of patients with RA initiating biologics demonstrated that the unadjusted probability of TCZ persistence, defined as time to therapy switch or discontinuation, was $51.5 \% 1$ year after initiation and $38.8 \% 2$ years after initiation [5]. It is also important to note that the present study analyzed durability of response among patients who achieved MCID or LDA, whereas other studies examined the persistence among all patients; this needs to be taken into account when comparing these findings.

To better inform treatment decisions for patients with RA, baseline characteristics were analyzed to determine if they were associated with durability of TCZ therapy. Our analysis indicated that higher baseline CDAI score and 
A

Duration of RA (5-year effect)
CDAl (5-unit effect)
Age (5-year effect)
History of diabetes
History of malignancy
Prior non-TNFi use (ref = 0 non-TNFis)
$\quad 1$ non-TNFi
$\geq 2$ non-TNFis

All TCZ Initiators

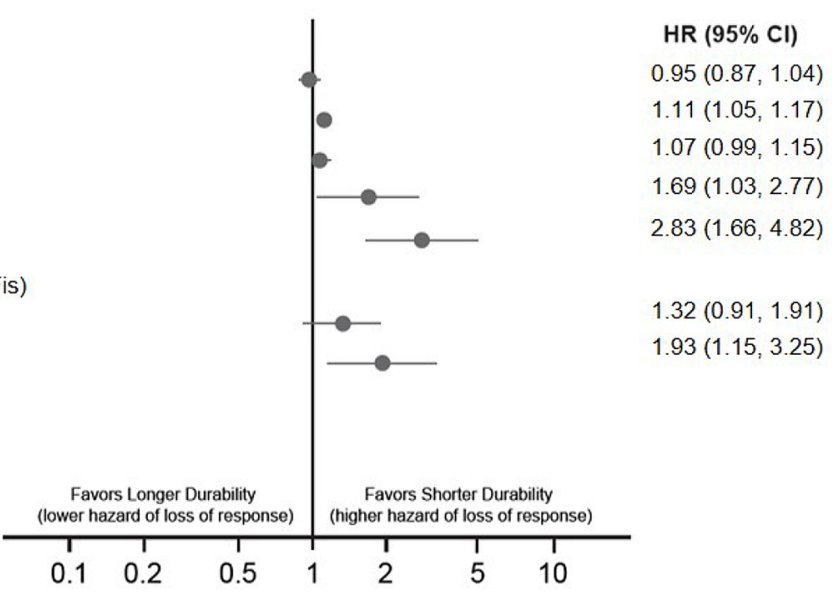

TCZ-IV Initiators

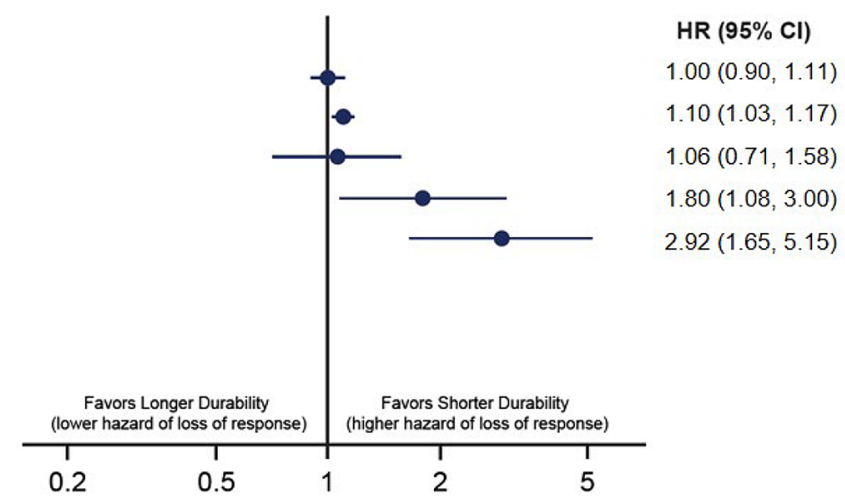

TCZ Initiators Excluding Those with No Reported Reason for Discontinuation

Duration of RA (5-year effect) CDAI (5-unit effect)

Age (5-year effect)

History of malignancy

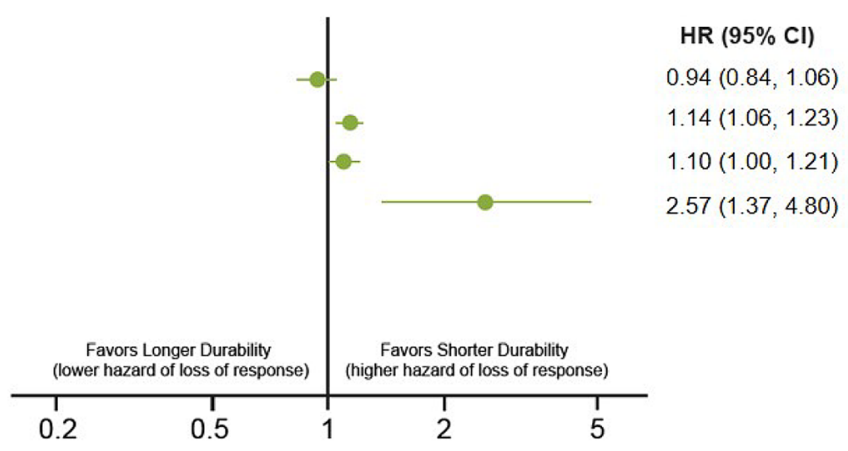

Fig. 5 Estimated effects of covariates on LDA durability of response. $C D A I$ Clinical Disease Activity Index, $I V$ intravenous, $L D A$ low disease activity, $R A$ rheumatoid arthritis, Ref reference, $T C Z$ tocilizumab, TNFi tumor necrosis factor inhibitor

duration of RA were associated with longer MCID durability, and no factors associated with longer LDA durability were identified. Perhaps this association between higher baseline disease activity and MCID improvement may be explained by the fact that it is feasible to have 
greater improvement with higher baseline disease activity. Higher baseline CDAI score was also associated with shorter LDA durability. Since LDA is a more stringent criterion of response than MCID, it is to be expected that these two measures of disease activity would not have the same association with baseline CDAI score. It is likely easier to achieve and maintain MCID than LDA. For example, if a patient's CDAI score improves from 30 to 15 , they have achieved MCID but not LDA. Furthermore, a patient could achieve LDA at one visit but at the next visit their CDAI score could increase slightly, which puts them outside the range for achieving LDA but still within range for MCID. Thus, for patients with higher baseline CDAI scores, maintenance of MCID may be more achievable than maintenance of LDA.

Shorter durability was associated with history of malignancy and history of diabetes in analyses of both MCID and LDA durability; however, these represented small subgroups of the overall study population (6.1 and $10.3 \%$, respectively), and the underlying reasons for these associations are not clear. Studies have shown that treatment with TCZ is not associated with malignancy or diabetes. In a post hoc analysis of five phase 3 trials and long-term extension studies of TCZ in patients with RA, adjudicated malignancy rates among those who received TCZ with or without methotrexate or DMARDs were not greater than rates among those who received placebo and methotrexate or DMARDs; in the TCZ all-exposure population, rates remained consistent over time [25]. Patients with RA receiving TCZ treatment were shown to have lower glycated hemoglobin A1c levels after TCZ initiation, and treatment with TCZ was found to improve insulin sensitivity $[26,27]$. Shorter LDA durability was also associated with higher baseline CDAI score.

This study has some limitations inherent to observational studies. The generalizability of the data to broader patient populations is a potential limitation. However, patients from diverse backgrounds are represented in the Corrona RA Registry, as there are participating practices in academic, private, rural, and urban settings. Additionally, generalizability of the registry data has been supported by a study showing that among US patients with RA covered by Medicare, baseline characteristics of patients in the Corrona RA Registry were comparable to those of the general population [18]. It is possible that patients could have intermittently been out of LDA or MCID between study visits. Use of the Corrona RA Registry enabled a large patient population to be included, which is a strength of this study.

\section{CONCLUSIONS}

In this real-world RA population who initiated TCZ, most patients received $\geq 2$ prior biologics. Median durability of response was $>3$ years when measured as maintenance of at least MCID in CDAI score while receiving therapy with TCZ; even with the more stringent criteria of maintenance of LDA, durability of response was $>1$ year. Understanding durability of response to TCZ may help inform treatment decisions for patients with RA.

\section{ACKNOWLEDGEMENTS}

Funding. This study was sponsored by Corrona LLC, and the analysis was funded by Genentech, Inc. Access to study data was limited to Corrona, and Corrona statisticians completed all of the analyses; all authors contributed to the interpretation of the results. Corrona has been supported through contracted subscriptions in the last 2 years by AbbVie, Amgen, Boehringer Ingelheim, Bristol Myers Squibb, Celgene, Crescendo, Lilly, Genentech, Gilead, GSK, Janssen, Merck, Momenta, Novartis, Ortho Dermatologics, Pfizer, Regeneron, Roche, Sanofi, and Sun. The study sponsor also funded the journal's Rapid Service Fee.

Medical Writing Assistance Support for thirdparty writing assistance, provided by Claire Stedden, PhD, of Health Interactions, Inc, was provided by Genentech, Inc. 
Authorship All named authors meet the International Committee of Medical Journal Editors (ICMJE) criteria for authorship for this article, take responsibility for the integrity of the work as a whole, and have given their approval for this version to be published.

Prior Presentation A portion of this work was presented at the European League Against Rheumatism (EULAR) E-Congress of Rheumatology, June 2020.

Disclosures Dimitrios A. Pappas is an employee and shareholder of Corrona, LLC, and a consultant for Regeneron, Novartis, and Roche. Taylor Blachley and Kelechi Emeanuru are employees of Corrona, LLC. Jennie H. Best and William G. Reiss are employees and shareholders of Genentech, Inc. Steve Zlotnick was an employee of Genentech, Inc., at the time of this study and is shareholder of Genentech, Inc. Joel M. Kremer is president of the Corrona Research Foundation, founder of Corrona, LLC, and a consultant for AbbVie, Amgen, Bristol Myers Squibb, Genentech, Gilead, GSK, Lilly, Pfizer, Regeneron, and Sanofi. Article processing charges were funded by Genentech, Inc.

Compliance with Ethics Guidelines Ethics approvals for this study were obtained from a central institutional review board (IRB; New England Independent Review Board 120160610). For academic investigative sites that did not receive a waiver to use the central IRB, full board approval was obtained from the respective governing IRBs, and documentation of approval was submitted to the sponsor prior to initiating any study procedures. All procedures performed in studies involving human participants were in accordance with the ethical standards of the institutional and/or national research committee and with the 1964 Helsinki Declaration and its later amendments or comparable ethical standards. Informed consent was obtained from all individual participants included in the study.

Data Availability The data sets generated and analyzed during the current study are not publicly available because of proprietary rights but are available from the corresponding author on reasonable request.

Open Access. This article is licensed under a Creative Commons Attribution-NonCommercial 4.0 International License, which permits any non-commercial use, sharing, adaptation, distribution and reproduction in any medium or format, as long as you give appropriate credit to the original author(s) and the source, provide a link to the Creative Commons licence, and indicate if changes were made. The images or other third party material in this article are included in the article's Creative Commons licence, unless indicated otherwise in a credit line to the material. If material is not included in the article's Creative Commons licence and your intended use is not permitted by statutory regulation or exceeds the permitted use, you will need to obtain permission directly from the copyright holder. To view a copy of this licence, visit http://creativecommons.org/licenses/by$\mathrm{nc} / 4.0 /$.

\section{REFERENCES}

1. Aletaha D, Neogi T, Silman AJ, et al. 2010 Rheumatoid arthritis classification criteria: an American College of Rheumatology/European League Against Rheumatism collaborative initiative. Arthritis Rheum. 2010;62(9):2569-81.

2. Smolen JS, Landewé RBM, Bijlsma JWJ, et al. EULAR recommendations for the management of rheumatoid arthritis with synthetic and biological disease-modifying antirheumatic drugs: 2019 update. Ann Rheum Dis. 2020;79(6):685-99.

3. Nam JL, Takase-Minegishi K, Ramiro S, et al. Efficacy of biological disease-modifying antirheumatic drugs: a systematic literature review informing the 2016 update of the EULAR recommendations for the management of rheumatoid arthritis. Ann Rheum Dis. 2017;76(6):1113-36.

4. Murage MJ, Tongbram V, Feldman SR, et al. Medication adherence and persistence in patients with rheumatoid arthritis, psoriasis, and psoriatic arthritis: a systematic literature review. Patient Prefer Adherence. 2018;12:1483-503.

5. Johnston SS, McMorrow D, Farr AM, Juneau P, Ogale S. Comparison of biologic disease-modifying antirheumatic drug therapy persistence between 
biologics among rheumatoid arthritis patients switching from another biologic. Rheumatol Ther. 2015;2(1):59-71.

6. ACTEMRA Prescribing Information. Genentech, Inc. South San Francisco, CA. 2020

7. Smolen JS, Beaulieu A, Rubbert-Roth A, et al. Effect of interleukin-6 receptor inhibition with tocilizumab in patients with rheumatoid arthritis (OPTION study): a double-blind, placebo-controlled, randomised trial. Lancet. 2008;371(9617):987-97.

8. Genovese MC, McKay JD, Nasonov EL, et al. Interleukin-6 receptor inhibition with tocilizumab reduces disease activity in rheumatoid arthritis with inadequate response to disease-modifying antirheumatic drugs: the tocilizumab in combination with traditional disease-modifying antirheumatic drug therapy study. Arthritis Rheum. 2008;58(10): 2968-80.

9. Emery P, Keystone E, Tony HP, et al. IL-6 receptor inhibition with tocilizumab improves treatment outcomes in patients with rheumatoid arthritis refractory to anti-tumour necrosis factor biologicals: results from a 24-week multicentre randomised placebo-controlled trial. Ann Rheum Dis. 2008;67(11):1516-23.

10. Jones G, Sebba A, Gu J, et al. Comparison of tocilizumab monotherapy versus methotrexate monotherapy in patients with moderate to severe rheumatoid arthritis: the AMBITION study. Ann Rheum Dis. 2010;69(1):88-96.

11. Kremer JM, Blanco R, Brzosko M, et al. Tocilizumab inhibits structural joint damage in rheumatoid arthritis patients with inadequate responses to methotrexate: results from the double-blind treatment phase of a randomized placebo-controlled trial of tocilizumab safety and prevention of structural joint damage at one year. Arthritis Rheum. 2011;63(3):609-21.

12. Burmester GR, Rubbert-Roth A, Cantagrel A, et al. A randomised, double-blind, parallel-group study of the safety and efficacy of subcutaneous tocilizumab versus intravenous tocilizumab in combination with traditional disease-modifying antirheumatic drugs in patients with moderate to severe rheumatoid arthritis (SUMMACTA study). Ann Rheum Dis. 2014;73(1):69-74.

13. Kivitz A, Olech E, Borofsky M, et al. Subcutaneous tocilizumab versus placebo in combination with disease-modifying antirheumatic drugs in patients with rheumatoid arthritis. Arthritis Care Res. 2014;66(11):1653-61.

14. Kremer JM, Rigby W, Singer NG, et al. Sustained response following discontinuation of methotrexate in patients with rheumatoid arthritis treated with subcutaneous tocilizumab: results from a randomized controlled trial. Arthritis Rheumatol (Hoboken). 2018;70(8):1200-8.

15. Kremer JM, Blanco R, Halland AM, et al. Clinical efficacy and safety maintained up to 5 years in patients with rheumatoid arthritis treated with tocilizumab in a randomised trial. Clin Exp Rheumatol. 2016;34(4):625-33.

16. Kivitz A, Wallace T, Olech E, et al. Long-term safety and efficacy of subcutaneously administered tocilizumab for adult rheumatoid arthritis: a multicenter phase $3 \mathrm{~b}$ long-term extension study. Rheumatol Ther. 2016;3(2):291-304.

17. Kremer JM. The Corrona US registry of rheumatic and autoimmune diseases. Clin Exp Rheumatol. 2016;34(5 Suppl 101):S96-9.

18. Curtis JR, Chen L, Bharat A, et al. Linkage of a deidentified United States rheumatoid arthritis registry with administrative data to facilitate comparative effectiveness research. Arthritis Care Res (Hoboken). 2014;66(12):1790-8.

19. Curtis JR, Yang S, Chen L, et al. Determining the minimally important difference in the clinical disease activity index for improvement and worsening in early rheumatoid arthritis. Arthritis Care Res. 2015;67(10):1345-53.

20. Lauper K, Nordstrom DC, Pavelka K, et al. Comparative effectiveness of tocilizumab versus TNF inhibitors as monotherapy or in combination with conventional synthetic disease-modifying antirheumatic drugs in patients with rheumatoid arthritis after the use of at least one biologic diseasemodifying antirheumatic drug: analyses from the pan-European TOCERRA register collaboration. Ann Rheum Dis. 2018;77(9):1276-82.

21. Gabay C, Riek M, Hetland ML, et al. Effectiveness of tocilizumab with and without synthetic diseasemodifying antirheumatic drugs in rheumatoid arthritis: results from a European collaborative study. Ann Rheum Dis. 2016;75(7):1336-42.

22. Harrold LR, Reed GW, John A, et al. Cluster-randomized trial of a behavioral intervention to incorporate a treat-to-target approach to care of US patients with rheumatoid arthritis. Arthritis Care Res (Hoboken). 2018;70(3):379-87.

23. Zak A, Corrigan C, Yu Z, et al. Barriers to treatment adjustment within a treat to target strategy in rheumatoid arthritis: a secondary analysis of the TRACTION trial. Rheumatology (Oxford). 2018;57(11):1933-7. 
24. Lau AN, Thorne JC, Movahedi M, et al. Effect of concomitant disease-modifying antirheumatic drugs and methotrexate administration route on biologic treatment durability in rheumatoid arthritis: OBRI cohort results. J Rheumatol. 2019;46(8):874-86.

25. Rubbert-Roth A, Sebba A, Brockwell L, et al. Malignancy rates in patients with rheumatoid arthritis treated with tocilizumab. RMD Open. 2016;2(1): e000213.
26. Otsuka Y, Kiyohara C, Kashiwado Y, et al. Effects of tumor necrosis factor inhibitors and tocilizumab on the glycosylated hemoglobin levels in patients with rheumatoid arthritis; an observational study. PLoS ONE. 2018;13(4):e0196368.

27. Schultz O, Oberhauser F, Saech J, et al. Effects of inhibition of interleukin-6 signalling on insulin sensitivity and lipoprotein (a) levels in human subjects with rheumatoid diseases. PLoS ONE. 2010;5(12):e14328. 